\title{
Endoscopic Endonasal Approach for Resection of a Pediatric Craniopharyngioma: Operative Video and Technical Nuances
}

\author{
James K. Liu'1,2,3 Jean Anderson Eloy 1,2,3,4
}

\footnotetext{
${ }^{1}$ Department of Neurological Surgery, Neurological Institute of New Jersey, Rutgers University, New Jersey Medical School, Newark, New Jersey, United States

2 Department of Otolaryngology-Head and Neck Surgery, Neurological Institute of New Jersey, Rutgers University, New Jersey Medical School, Newark, New Jersey, United States

${ }^{3}$ Center for Skull Base and Pituitary Surgery, Neurological Institute of New Jersey, Rutgers University, New Jersey Medical School, Newark, New Jersey, United States

${ }^{4}$ Department of Ophthalmology and Visual Science, Neurological Institute of New Jersey, Rutgers University, New Jersey Medical School, Newark, New Jersey, United States
}

Address for correspondence James K. Liu, MD, Department of Neurological Surgery, Rutgers University, New Jersey Medical School, 90 Bergen Street, Suite 8100, Newark, New Jersey 07103, United States (e-mail: james.liu.md@rutgers.edu).

J Neurol Surg B 2018;79(suppl S3):S245-S246.

\begin{abstract}
We present a pediatric case of a retrochiasmatic craniopharyngioma in the suprasellar region with third ventricular extension that was resected through a purely endoscopic endonasal approach (EEA) via the transplanum transtuberculum corridor. The patient is a 12-year-old boy who presented with progressive visual loss and panhypopituitarism. The EEA allows direct visualization of the undersurface of the optic chiasm and hypothalamus so that safe and meticulous tumor dissection can be performed to preserve these critical neurovascular structures. This video atlas demonstrates the operative technique and surgical nuances of the endoscopic skull base approach,

Keywords

- endoscopic endonasal approach

- craniopharyngioma

- pediatric skull base surgery

- suprasellar tumor microdissection of the tumor from the critical neurovascular structures, and multilayered reconstruction of the skull base defect with a nasoseptal flap. A gross total resection was achieved, and the patient was neurologically intact with improved visual acuity and visual fields. In summary, the EEA via the transplanum transtuberculum corridor is an important strategy in the armamentarium for surgical management of pediatric craniopharyngiomas.

The link to the video can be found at: https://youtu.be/bmgO_PMRHPk.
\end{abstract}

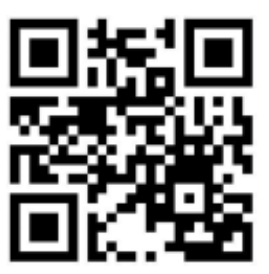

received

December 24, 2017

accepted

January 2, 2018

published online

February 26, 2018 www.thieme.com/skullbasevideos

www.thieme.com/jnlsbvideos
(C) 2018 Georg Thieme Verlag KG Stuttgart · New York

License terms

10.1055/s-0038-1626707. ISSN 2193-6331. 


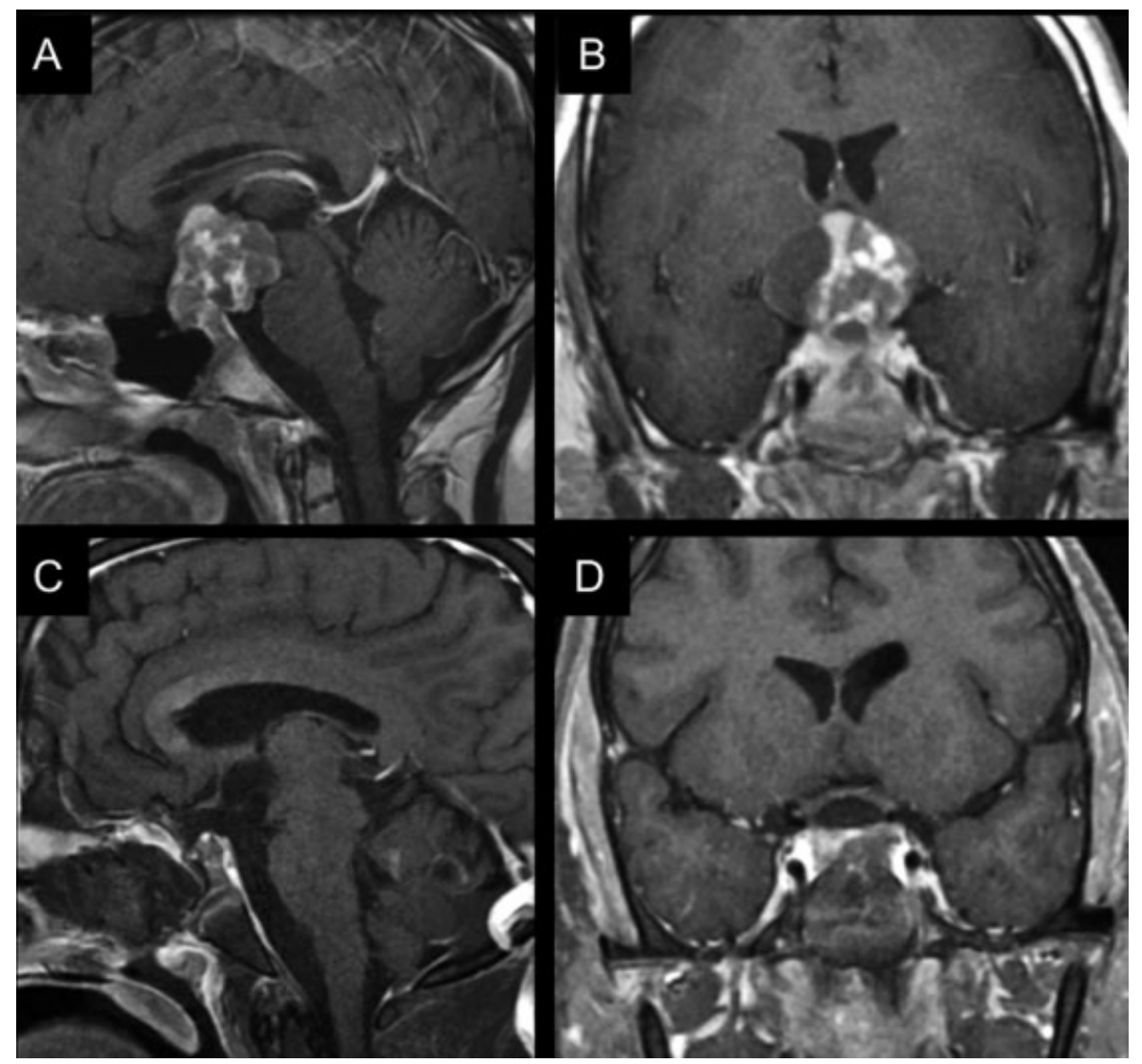

Fig. 1 Preoperative (A: sagittal, B: coronal) and postoperative (C: sagittal, D: coronal) postgadolinium T1-weighted MRI of a retrochiasmatic craniopharyngioma removed via an endoscopic endonasal approach. Gross total resection was achieved. MRI, magnetic resonance imaging.

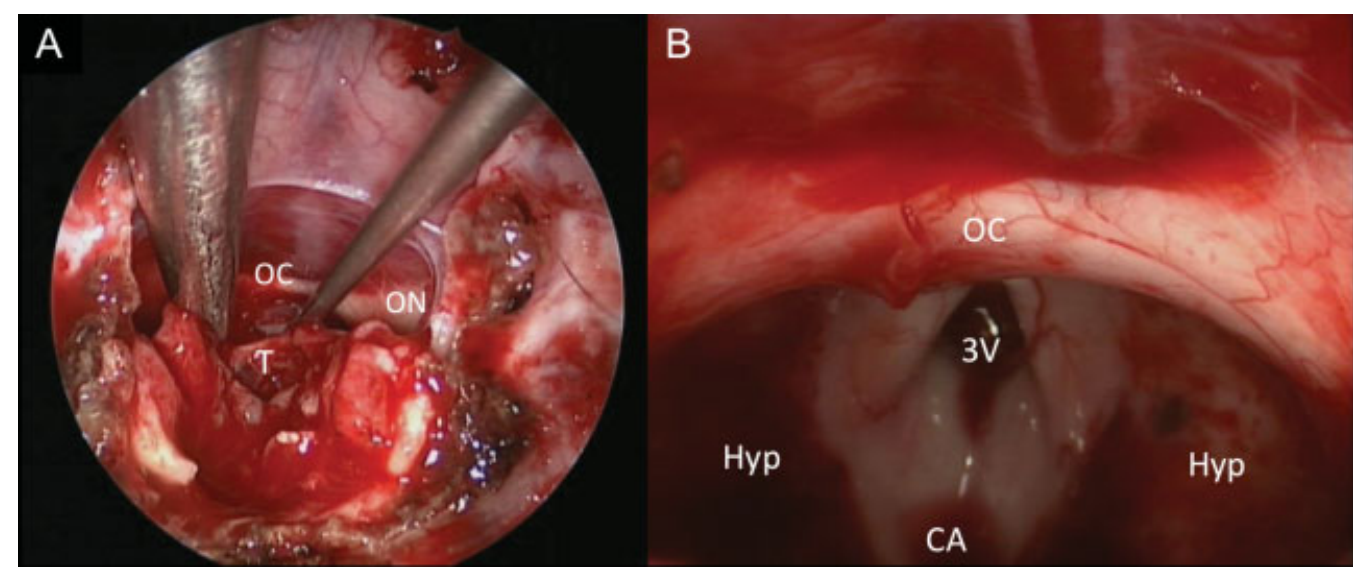

Fig. 2 (A) Intraoperative endoscopic view of the tumor ( $T$ ) being dissected from the undersurface of the optic chiasm (OC) and left optic nerve (ON). (B) View of the resection bed after total removal of the tumor. 3V, third ventricle; CA, cerebral aqueduct; Hyp, hypothalamus. 